\title{
Determinantes da posse de telefonia móvel no Brasil: o acesso à internet importa?
}

\author{
Marislei Nishijima1, Jorge Fagundes ${ }^{2}$, Marcos A. M. Lima ${ }^{3}$ \\ ${ }^{1}$ Escola de Artes, Ciências e Humanidades - Universidade de São Paulo \\ ${ }^{2}$ Universidade Federal do Rio de Janeiro \\ ${ }^{3}$ Pontifícia Universidade Católica do Rio de Janeiro \\ \{marislei\}Cusp.br, \{fagundes,mlima\} @fagundesconsultoria.com.br
}

\begin{abstract}
Since 2008 access to the Internet has become mandatory in Brazilian mobile telephony. This paper investigates the determinants of ownership of this segment using PNAD survey data from 2008 to 2009. The results, using logit model, show that Internet access increases the probability of ownership of mobile telephony in $19 \%$.
\end{abstract}

Resumo. Desde 2008 o acesso à internet passou a ser obrigatório na telefonia móvel brasileira. Este artigo investiga quais são os determinantes da posse de telefonia móvel no Brasil a partir dos microdados da PNAD referente aos anos de 2008 e 2009. Os resultados, obtidos a partir de modelos logit, mostram que o acesso à internet aumenta a probabilidade de posse de telefonia móvel em $19 \%$.

\section{Introdução}

O Brasil, assim como os demais países em desenvolvimento, constitui atualmente um dos principais consumidores novos de telefonia móvel. Estes países foram responsáveis, para o período entre 2004 e 2009, por $80 \%$ de novas linhas móveis [Khalil e Kenny, 2008]. Nestes países, conforme Khalil e Kenny (2008), estes novos usuários, principalmente em telefonia móvel pré-paga, estão relacionados com a ampliação dos serviços disponíveis para comunicação e informação e com expansão de serviços bancários e de crédito para as classes de renda mais baixa.

A Tabela 1, com informações do Banco Mundial, (WDI) ${ }^{1}$, resume o padrão de evolução do grau de cobertura da telefonia móvel do Brasil em relação à Colômbia e aos Estados Unidos até o ano de 2007.

Tabela 1 - Percentual da População com Cobertura de Rede de Telefonia Móvel

\begin{tabular}{|l|c|c|c|}
\hline \multicolumn{3}{|c|}{ População com cobertura de rede de telefonia móvel (\%) } \\
\hline Ano & Brasil & Colombia & Estados Unidos \\
\hline 2004 & 88 & 80 & 99 \\
\hline 2005 & 88.3 & 82 & 99 \\
\hline 2006 & 89.4 & 83 & 99.8 \\
\hline
\end{tabular}

\footnotetext{
${ }^{1}$ World Development Indicators (WDI) of World Bank: http://data.worldbank.org/indicator.
} 


\begin{tabular}{|l|c|c|c|}
\hline 2007 & 90.6 &.. & 99.8 \\
\hline
\end{tabular}

Considerando a possibilidade de acesso à internet por meio da telefonia móvel, a ampliação do mercado de telefonia móvel em direção às classes mais pobres deve implicar numa ampliação do acesso dessas classes à rede mundial de informações e conseqüentemente aos seus serviços e mercados [Spence e Smith, 2010].

Deste modo, o objetivo deste estudo é estimar os determinantes da posse de telefonia móvel para o Brasil com vistas a obter informações mais precisas sobre variáveis que afetam a decisão de posse de um telefone móvel por parte dos consumidores. Em especial, verificar se o acesso à internet é significativo para explicar a posse de telefone móvel.

O conhecimento destes determinantes permite uma melhor avaliação sobre a decisão de consumo das famílias e podem balizar decisões de empresas de telefonia de negócios na internet. Principalmente porque desde 2008, com plano de término em 2010, o serviço de internet está disponível para a telefonia celular no Brasil por regulação da ANATEL ${ }^{2}$, conforme portaria 178/2008 ${ }^{3}$. Esse potencial aumento de acesso à internet por telefonia móvel poderá aumentar em grande escala a demanda por serviços de sistemas de informação associados a este veículo de comunicação e as empresas envolvidas poderão balizar suas decisões iniciais nos resultados obtidos.

A maioria dos estudos que investiga os determinantes da posse de telefonia móvel estima demandas e tem como enfoque a discussão sobre substituição/complementariedade entre telefonia móvel e fixa e não exatamente o perfil do consumidor [Ward e Zheng 2011; Gupta e Jain 2011; e Afridi et al. 2011]. Rodini et al. (2002) estimam a função de demanda por telefonia celular e pela segunda linha da telefonia fixa, através de uma função logit, utilizando microdados, para os anos 2000 e 2001, em 294 áreas urbanas nos Estados Unidos.

Considerando as limitações das informações disponíveis na PNAD (Pesquisa Nacional de Amostra por Domicílio do Instituto Brasileiro de Geografia e Estatística-IBGE), que não possui informações sobre preços, procede-se ao estudo dos determinantes da posse de telefonia móvel.

O artigo apresenta 4 seções além da introdução. A seção 2 discute a estratégia metodológica e a 3 apresenta os dados utilizados. Na seção 4 são apresentados os resultados e, por fim, a seção 5 apresenta as conclusões.

\section{Estratégia metodológica}

A PNAD possuir informação sobre posse de aparelhos telefônicos móveis, mas não discrimina a quantidade utilizada destes serviços por cada indivíduo. Assim, estimou-se a probabilidade de posse de telefonia móvel a partir de modelos logit, conforme Ida e Kuroda (2009) e Schmitd (2005). A equação básica que descreve o modelo de escolha deste tipo de serviço é mostrada abaixo. Note que esta equação decorre de um modelo econômico de um agente $i$ que obtém utilidade, representada por $U_{i}($.$) , a partir do$ consumo dos bens [Mas-Collel et al. 1995].

\footnotetext{
${ }^{2}$ Agência Nacional de Telecomunicações.

${ }^{3}$ www.anatel.gov.br
} 


$$
P_{i}=\operatorname{Pr} o b\left[U_{i}\left(y_{i}=1\right)>U_{i}\left(y_{i}=0\right)\right]
$$

O modelo estatístico estimado pode ser descrito da seguinte forma:

$$
y_{i}^{*}=\beta_{0}+\sum_{j=1}^{k} \beta_{j} X_{i j}+u_{i}
$$

Sendo que $y^{*}{ }_{i}$ é uma variável latente não observada, que descreve a probabilidade do indivíduo $i$ consumir um aparelho de telefone. Assim utilizamos a parte da informação de $y_{i}$ que pode ser observada, 1 se o indivíduo possui aparelho com linha telefônica e 0 caso contrário e, usando alguma distribuição estatística (neste caso, a logística), é obtida esta variável latente, que apresenta valor entre zero e um. O índice $j$ relaciona as diferentes variáveis observadas que explica/afetam a probabilidade de posse de aparelhos telefônicos, em acordo com a teoria econômica. $\beta_{j}$ são os coeficientes a serem estimados. A variável residual aleatória $u_{i}$ descreve choques individuais e possui média zero e segue uma distribuição logística [Wooldridge 2002].

A variável binária observada $y_{i}$ pode ser definida da seguinte maneira:

$$
y_{i}=1 \text { se } y^{*}>0 \text { ou } U(y=1)>U(y=0) \quad(3) ; \quad y_{i}=0 \text { se } y^{*}>0 \text { ou } U(y=1) \leq U(y=0)
$$

Onde $U_{i}$ representa a utilidade do indivíduo $i$ ter um aparelho telefônico $U_{i}\left(y_{i}=1\right)$ ou não ter $U_{i}\left(y_{i}=0\right)$. O indivíduo consumirá o bem, $y=1$, caso sua utilidade seja com o consumo seja maior do que usar a renda com outro bem.

Considere $P_{i}$ a probabilidade de ocorrência do evento possuir telefone e ( $1-P_{i}$ ) sua não ocorrência, então a partir de (2) a (4), pode-se descrever a probabilidade de ocorrência do evento como sendo:

$$
\mathrm{P}_{\mathrm{i}}=\operatorname{Prob} \text { ob }\left(\mathrm{y}_{\mathrm{i}}=1\right)=\operatorname{Prob} \text { ob }\left[\mathrm{u}_{\mathrm{i}}>-\mathrm{z}_{\mathrm{i}}\right]=1-\mathrm{F}\left[-\mathrm{z}_{\mathrm{i}}\right]
$$

Sendo $z_{i}=\beta_{0}+\sum_{j=1}^{k} \beta_{j} X_{i j}$ e $F($.$) a função de distribuição acumulada de u_{i}$ com forma funcional logística expressa por: $F\left(z_{i}\right)=\frac{\exp \left(z_{i}\right)}{1+\exp \left(z_{i}\right)}$

Como $F\left(z_{i}\right)=1-F\left(z_{i}\right)$, pode-se mostrar que $\quad P_{i}=\frac{\exp \left(z_{i}\right)}{1+\exp \left(z_{i}\right)}$

Dessa forma, é estimado modelo tendo como variável dependente $\mathrm{P}_{\mathrm{i}}$ a posse de telefone móvel e como variáveis independentes características sócio-econômicas incluindo o acesso à internet em período recente.

\section{Dados}

Foram utilizados os microdados da PNADs de 2008 e de 2009, na faixa etária de 10 a 60 anos, que entrevista aproximadamente 80 mil famílias $^{4}$ em todo Brasil nos anos em que não ocorre o CENSO. No questionário existe a pergunta sobre a posse de telefonia

\footnotetext{
${ }^{4}$ Cada família tem em média 4 pessoas, resultando em informações sobre 320 mil pessoas por ano
} 
móvel (celular e rádio freqüência) para o indivíduo. Além disso, existem informações sobre domicílios que inclui a posse ou não de linhas telefônicas fixas.

As variáveis demográficas, descritivas do padrão de vida e do perfil dos indivíduos utilizados na estimação foram as seguintes: i) acesso à Internet (internet); ii) sexo (mascu); iii) idade; iv) anos de estudo (estudo); v) economicamente ativo (pea); vi) raça (racad1 - racad6); vii) condição do indivíduo na família (cond_fa_d1 - cond_fa_d8); viii) renda (renda_individual); ix) urbano (urbana); x) tipo de ocupação (ocupacao_d1 ocupação_d12); xi) posse de telefonia fixa (fixo); xii) computador (pc). Além destas variáveis, foram utilizadas variáveis binárias (dummies) de grandes regiões do país, de unidade da federação de residência e de ano para captar efeitos macroeconômicos.

Em relação à variável dependente, variável binária, observa-se que varia de acordo com a idade dos indivíduos. A Figura 1 mostra o percentual de pessoas que possui telefonia móvel conforme a idade medida em anos. Mostra também o percentual de pessoas que possui telefonia fixa. Verifica-se que a posse do celular é mais alta em termos percentuais entre 14 e 55 anos. No caso da telefonia fixa, o padrão é levemente crescente com a idade.

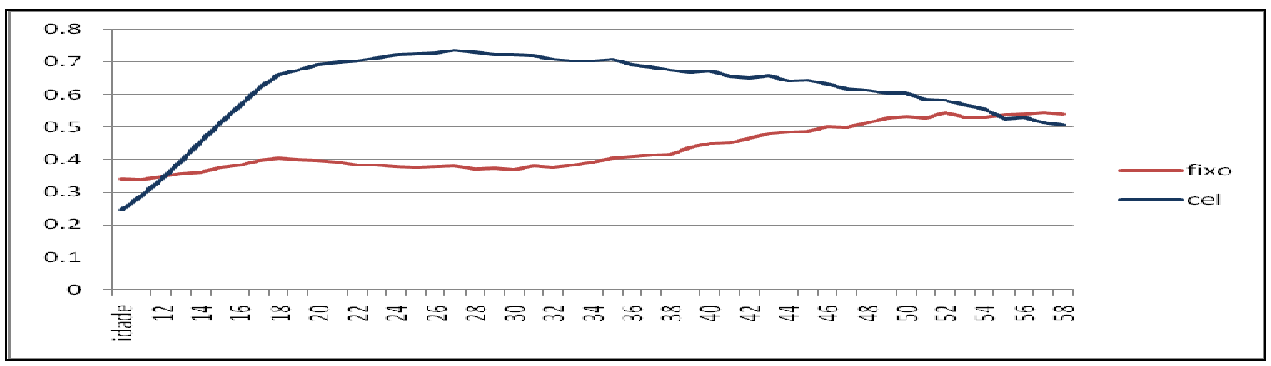

Figura 1: Percentual de pessoas que possui telefone fixo e móvel conforme idade a partir da PNAD de 2009

Além disso, a análise destas variáveis, de acordo com a faixa de renda dos indivíduos, mostra, conforme medianas, que a posse de telefone móvel está associada com renda média baixa, $\mathrm{R} \$ 487,00$ mensais.

No Brasil, de acordo com as PNADs de 2008 e 2009, uma parcela maior da população na faixa etária entre 10 e 60 anos, 61,8\%, possui aparelhos de telefonia móvel comparado com a parcela do grupo que possui telefonia fixa, que corresponde a $41,9 \%$.

\section{Resultados e Discussões}

Os resultados obtidos com as estimativas logit encontram-se na tabela 2. Os valores reportados são os coeficientes estimados. Verifica-se que o fato do indivíduo ter acessado a internet nos últimos meses aumenta a sua probabilidade de possuir um telefone móvel. A idade afeta positivamente a probabilidade de posse e seu avanço, avaliado pela idade ao quadrado, ocorre a taxas decrescentes. Entretanto, o efeito marginal é pequeno. $\mathrm{O}$ mesmo fenômeno acontece com a renda mensal individual. $\mathrm{O}$ tamanho da população, a escolaridade, o fato do indivíduo pertencer à população economicamente ativa (PEA), ter telefone fixo, morar em zona urbana e ter computador afetam positivamente a probabilidade de posse de telefone móvel.

Os resultados das dummies de raça ( $\mathrm{racad}$ ), ocupação (ocupacaod), condição na família (cond_fam_d) e grande região (regiaod) devem ser lidos respectivamente em relação a 
brancos, empregado permanentes em serviços auxiliares, chefe de família e região sudeste. Assim, verifica-se que: a raça ou etnia dos indivíduos não afeta a probabilidade de posse de maneira diferenciada; que ser empregado formal permanente em serviços auxiliares, chefe de família e morar nas regiões Sul e Sudeste aumentam a probabilidade de posse de telefonia móvel.

A Tabela 3 mostra os efeitos marginais calculados para os coeficientes estimados. Estes valores mostram qual a variação na probabilidade de possuir um telefone móvel levando em conta as características apresentadas. Verifica-se que o fato do indivíduo ter acessado a internet nos últimos 2 meses, seja o acesso onde for (computador, celular, tablet), aumenta sua probabilidade de possuir telefone móvel em 19\%. Assim como ser do sexo masculino reduz em $7 \%$ sua chance de posse e assim sucessivamente.

\section{Tabela 2: Resultados dos coeficientes estimados}

\begin{tabular}{|c|c|c|c|c|c|}
\hline VARIABLES & telefone móvel & descriçao & VARIABLES & telefone móvel & descriçao \\
\hline \multirow[t]{2}{*}{ internet } & $0.921^{\star \star *}$ & \multirow{2}{*}{$\begin{array}{c}\text { acesso à internet nos últimos } 2 \\
\text { meses }\end{array}$} & \multirow[t]{2}{*}{ cond_fa_d2 } & $-0.298^{\star \star \star}$ & \multirow{2}{*}{ cônjugue } \\
\hline & $(0.00942)$ & & & $(0.00973)$ & \\
\hline \multirow{2}{*}{ mascu } & $-0.320^{\star \star \star}$ & \multirow{2}{*}{ sexo masculino } & \multirow{2}{*}{ cond_fa_d3 } & $-0.283^{* \star *}$ & \multirow{2}{*}{ filho } \\
\hline & $(0.00800)$ & & & $\begin{array}{ll}(0.0116) \\
\end{array}$ & \\
\hline \multirow{2}{*}{ idade } & $0.115^{* * *}$ & \multirow{2}{*}{ idade } & \multirow{2}{*}{ cond_fa_d4 } & $-0.347^{* \star *}$ & \multirow{2}{*}{ outro parente } \\
\hline & $(0.00172)$ & & & $(0.0161)$ & \\
\hline \multirow{2}{*}{ idade2 } & $-0.00162^{* * *}$ & \multirow{2}{*}{ idade ao quadrado } & \multirow{2}{*}{ cond_fa_d5 } & $-0.250^{\star \star \star \star}$ & \multirow{2}{*}{ pensionista } \\
\hline & $(2.35 e-05)$ & & & $(0.0491)$ & \\
\hline \multirow{2}{*}{ estudo } & $0.118^{\star \star *}$ & \multirow{2}{*}{ anos de escolaridade } & \multirow{2}{*}{ cond_fa_d7 } & 1.288 & \multirow{2}{*}{ empregado doméstico } \\
\hline & $(0.00107)$ & & & $(1.215)$ & \\
\hline \multirow{2}{*}{ tam_fam } & $-0.0952^{\star \star *}$ & \multirow{2}{*}{$\begin{array}{l}\text { número de componentes que } \\
\text { moram no domicilio }\end{array}$} & \multirow{2}{*}{ ocupacaod2 } & -0.151 & \multirow{2}{*}{ empregado agricultura } \\
\hline & $(0.00192)$ & & & $(0.0962)$ & \\
\hline \multirow{2}{*}{ pop } & $1.91 \mathrm{e}-07^{\star \star \star *}$ & \multirow{2}{*}{ população do estado } & \multirow{2}{*}{ ocupacaod3 } & $-0.647^{\star \star \star}$ & empregado em outra \\
\hline & $(6.56 \mathrm{e}-08)$ & & & $(0.0218)$ & atividade \\
\hline nea & $0.342^{\star \star \star}$ & co individun nertencé PFA & 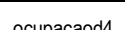 & $-0.349^{\star \star \star \star}$ & empreqadn temnorárin \\
\hline ped & $(0.0147)$ & Se murviduo perlence a reA & Oedpacdout4 & $(0.0129)$ & enpregado émporario \\
\hline renda individual & $0.000465^{\star \star \star}$ & renda mensal individual de todas & Ocunacand5 & 0.0131 & conta própria nos serviços \\
\hline renad_maniviaual & $(9.39 \mathrm{e}-06)$ & as fontes & ocupacaodos & $(0.0298)$ & auxiliares \\
\hline renda ind? & $-1.73 \mathrm{e}-08^{\star \star \star}$ & renda mensal individual ao & ocunacand6 & $-0.362^{\star \star \star}$ & conta nrónria pa arricultura \\
\hline renda_tma & $(5.21 \mathrm{e}-10)$ & quadrado & ocupacaodo & $(0.0185)$ & comila propria na agricuntura \\
\hline (nthong & $0.725^{\star \star *}$ & mesitêcis om z & 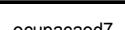 & $-0.194^{\star \star \star}$ & conta própria em outras \\
\hline uroana & $(0.0100)$ & residencla em zona uroana & ocupacaod $r$ & $(0.0127)$ & atividade \\
\hline fixo & $0.0731^{\star \star \star}$ & domicilin nocsui telefone fixo & ocunacand8 & $0.127^{* * \star}$ & empregador em serviços \\
\hline fixo & $(0.00820)$ & domicilio possui telefone fixo & ocupacaod8 & $(0.0321)$ & auxiliares \\
\hline & $0.578^{* \star \star}$ & domicilin mscui comnutador & Ocunacanda & $-0.815^{\star \star \star}$ & empregador na agricultura $\mathrm{e}$ \\
\hline $\mathrm{pc}$ & $(0.00905)$ & domicilio possui computador & ocupacaod9 & $(0.0276)$ & criação de animais \\
\hline (2) & 0.00389 & & & $-0.341^{* * *}$ & empregador em outras \\
\hline racad2 & $(0.0615)$ & cor preta & ocupacaod10 & $(0.123)$ & atividades \\
\hline $\operatorname{mach} 3$ & -0.0598 & cor amarela & Ocunasand11 & $-0.723^{\star \star \star}$ & trabalhador não remunerado \\
\hline racad3 & $(0.0623)$ & cor amarela & ocupacaod11 & $(0.0225)$ & membro de unidade \\
\hline & 0.0546 & & & $-0.330^{\star \star \star}$ & outro trabalhador não \\
\hline racad4 & $(0.0850)$ & cor parda & ocupacaod12 & $(0.0167)$ & remunerado \\
\hline & -0.0980 & & & $-1.051^{* \star *}$ & \\
\hline racad5 & $(0.0614)$ & indigena & regiaod1 & $(0.0632)$ & norte \\
\hline & -0.231 & cem declaracão & regiand? & $-2.099^{\star * *}$ & nordeste \\
\hline racad6 & $(0.163)$ & sem declaraçāo & regiaod2 & $(0.544)$ & nordeste \\
\hline & $0.0832^{* * *}$ & 2009 & & $-1.320^{\star \star \star}$ & \\
\hline anod2 & $(0.0103)$ & 2009 & regiaod5 & $(0.0807)$ & centro-oeste \\
\hline
\end{tabular}

Standard errors in parentheses: *** $\mathrm{p}<0.01, * * \mathrm{p}<0.05, * \mathrm{p}<0.1 . \mathrm{n}^{\circ}$ observações 556117.

Dummies de estados incluídas

Tabela 3: Resultados efeitos marginais

\begin{tabular}{|l|c|l|c|}
\hline variável & efeito marginal & Variável & efeito marginal \\
\hline internet & 0.194 & Pop & 0.000 \\
\hline mascu & -0.070 & Peã & 0.076 \\
\hline idade & 0.025 & renda_individual & 0.000 \\
\hline idade2 & 0.000 & renda_individual2 & 0.000 \\
\hline estudo & 0.026 & Urbana & 0.169 \\
\hline tam_fam & -0.021 & Fixo & 0.016 \\
\hline
\end{tabular}




\section{Conclusões}

Os resultados mostram que o acesso a internet é um fator significativo para explicar a posse de telefonia móvel no Brasil. O fato do indivíduo ter acessado a internet nos últimos 2 meses aumenta sua probabilidade de possuir telefone móvel em 19\%. Outros determinantes importantes que afetam positivamente a probabilidade de posse de telefonia móvel são o sexo feminino, a posse de computador, a condição de chefe de família, residir em zona urbana, ser empregado formal em serviços auxiliares e residir nas regiões sul e sudeste do país. A raça/etnia não afeta a probabilidade de posse de telefonia celular.

Levando em conta os possíveis efeitos da regulação, para a obrigatoriedade de acesso à internet na telefonia móvel, sobre potencial aumento de demanda em serviços de sistemas de informação, este estudo orienta ênfase em indivíduos já iniciados em internet, indivíduos do sexo feminino e participante do setor formal da economia como consumidores como o perfil do consumidor de serviços ofertados pela internet.

\section{Referências}

Afridi, S., Farooq, S., Ullah, M. e Rahmani, R. (2010) The Analysis of Cellular Services and Estimating Fixed to Mobile Price Elasticities - A Case Study of United Kingdom, European Journal of Scientific Research.

Ida, T., and Kuroda, T. (2009). Discrete choice model analysis of mobile telephone service demand in japan. Empirical Economics, 36(1), 65-80. doi:10.1007/s00181008-0187-0

Khalil, M. and Kenny, C. (2008) "The Next Decade of ICT Development: Access, Application and the forces of Convergence. Information Technologies and International Development, Vol. 4 (3), p. 1-6.

Gupta, R. and K. Jain (2011). Diffusion of mobile telephony in India: An empirical study. Technological Forecasting \& Social Change. Article in Press.

Mas-Colell, A., M Whinston, and J. Green (1995), Microeconomic Theory, Oxford University Press.

Rodini, M., Ward, M.R., Woroch, G.A. (2002). "Going Mobile: Substitutability Between Fixed and Mobile Access". Paper apresentado na Conferência "Competition in Wireless: Spectrum, Service and Technology Wars", Florida.

Schimitd, C. A. J. (2005). Três ensaios sobre concorrência em setores da economia brasileira. Tese de doutorado. Fundação Getúlio Vargas. Rio de Janeiro.

Spence, R. and Smith, M. L. (2010). "ICT Development, and Poverty Reduction: Five Emerging Stories". Information technologies and International Development, Vol.6, SE, Special Edition, p. 11-17.

Ward, M. R. and S. Zheng (2011). Mobile and Fixed Substitution for Telephone Service in China. Electronic copy available at: http://ssrn.com/abstract $=1822668$

Wooldridge, Jeffrey M. Econometric Analysis of Cross Section and Panel Data. Cambridge: The MIT Press, 2002. 